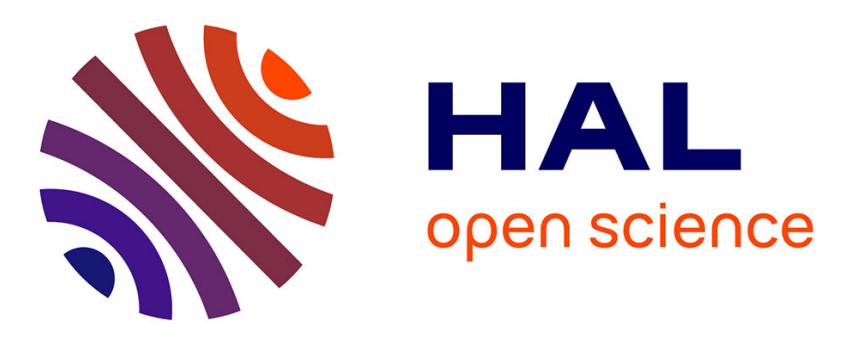

\title{
Additive effects of developmental acclimation and physiological syndromes on lifetime metabolic and water loss rates of a dry-skinned ectotherm
}

Mathias Dezetter, Andréaz Dupoué, Jean François Le Galliard, Olivier Lourdais

\section{To cite this version:}

Mathias Dezetter, Andréaz Dupoué, Jean François Le Galliard, Olivier Lourdais. Additive effects of developmental acclimation and physiological syndromes on lifetime metabolic and water loss rates of a dry-skinned ectotherm. Functional Ecology, 2022, 36 (2), pp.432-445. 10.1111/1365-2435.13951. hal-03405913

\section{HAL Id: hal-03405913 https://hal.science/hal-03405913}

Submitted on 9 Nov 2021

HAL is a multi-disciplinary open access archive for the deposit and dissemination of scientific research documents, whether they are published or not. The documents may come from teaching and research institutions in France or abroad, or from public or private research centers.
L'archive ouverte pluridisciplinaire HAL, est destinée au dépôt et à la diffusion de documents scientifiques de niveau recherche, publiés ou non, émanant des établissements d'enseignement et de recherche français ou étrangers, des laboratoires publics ou privés. 
1 Running title: determinant of lifetime metabolic and water loss rates of a dry-skinned ectotherm

\section{Additive effects of developmental acclimation and physiological}

\section{4 syndromes on lifetime metabolic and water loss rates of a dry-skinned}

\section{5 ectotherm}

6

7 Mathias Dezetter ${ }^{1,2, ~ *}$, Andréaz Dupoué ${ }^{1}$, Jean François Le Galliard ${ }^{1,3}$, Olivier Lourdais ${ }^{2,4}$

8

$9 \quad{ }^{1}$ CNRS Sorbonne Université, UMR 7618, iEES Paris, Université Pierre et Marie Curie, Tours 44-

10 45, 4 Place Jussieu, 75005 Paris, France

$11{ }^{2}$ Centre d'étude biologique de Chizé CNRS, UMR 7372, 79360, Villiers en Bois, France

$12{ }^{3}$ Ecole normale supérieure, PSL Research University, Département de biologie, CNRS, UMS 3194,

13 Centre de recherche en écologie expérimentale et prédictive (CEREEP-Ecotron IleDeFrance), 78

14 rue du château, 77140 Saint-Pierre-lès-Nemours, France

$15{ }^{4}$ School of Life Sciences, Arizona State University, Tempe, AZ 85287-4501, USA

$17 *$ Correspondence:

18 Mathias Dezetter, CEBC UMR 7372 CNRS-ULR

1979360 Villiers en Bois, FRANCE

20 Phone: $+33(0) 549093552$

21 Fax: + $33(0) 549096526$

22 E-mail: mathias.dezetter@gmail.com

23 ORCiD ID: 0000-0002-5083-624X

25 For consideration in: Functional Ecology

26 Figures: 4

27 Tables: 3 


\section{Acknowledgement}

29 The authors thank Maxime Le Henanff, Marie Vaugoyeau and Jérémie Souchet who contributed to 30 respirometry data acquisition and cares to vipers. The authors also thank Pierre de Villemereuil for

31 help with the construction and analysis of the bivariate model analysis using MCMCglmm. Funding 32 support was provided by the ANR JCJC ECTOCLIM, the Project AQUASTRESS 2018-1R20214 33 and Climate Sentinels Program 2016 - 2021 coordinated by the "Cistude Nature" association (PhD

34 Fanny Mallard) and funded by the European Regional Development Fund, the region Nouvelle

35 Aquitaine, the department of Gironde and Pyrénées-Atlantiques. M. D. is supported by a doctoral 36 grant from ED 227 Sorbonne University.

\section{Authors' contributions}

39 O.L. and A.D designed the study and collected the data. M.D. led the data analyses together with

40 O.L., J.-F.L.G. and A.D. M.D. led the writing of the manuscript and all authors contributed

41 critically in result interpretation and manuscript writing. All authors gave final approval for

42 publication.

\section{Conflict of interest}

45 The authors declare no competing or financial interests.

\section{Data accessibility}

48 Data will be deposited in Zenodo after acceptance. 


\section{Abstract}

51 1. Developmental plasticity and thermal acclimation can contribute to adaptive responses to climate

52 change by altering functional traits related to energy and water balance regulation. How plasticity

53 interacts with physiological syndromes through lifetime in long-lived species is currently unknown.

54 2. Here, we examined the impacts of long term thermal acclimation in a long-lived temperate

55 ectotherm (Vipera aspis) and its potential flexibility at adulthood for two related functional traits:

56 standard metabolic rate (SMR) and total evaporative water loss (TEWL).

57 3. We used climatic chambers to simulate three contrasted daily thermal cycles (warm, medium and

58 cold) differing in mean temperatures $\left(28,24\right.$, and $20^{\circ} \mathrm{C}$ respectively) and amplitudes $(5,10$ and

$5913^{\circ} \mathrm{C}$ respectively) during immature life ( 0 to 4 years of age). Individuals were then maintained

60 under common garden conditions (medium cycle) for an additional 3-years period (4 to 7 years of

61 age). SMR and TEWL were repeatedly measured in the same individuals throughout life during and

62 after the climate manipulation.

63 4. Individuals reduced their SMR (negative compensation) when experiencing the warm cycle but

64 flexibly adjusted their SMR to common garden conditions at adulthood. In addition, thermal

65 conditions during the juvenile life stage led to changes in TEWL persisting until adulthood.

66 5. We further found consistent intra-individual variation for SMR and TEWL and a positive intra-

67 individual and inter-individual covariation between them throughout life. Thus, plastic responses

68 were combined with a physiological syndrome linking SMR and TEWL.

69 6. Our study demonstrates the capacity of long-lived organisms to flexibly shift their SMR to reduce

70 daily maintenance costs in warmer and less variable thermal environments, which might be

71 beneficial for low energy specialist organisms such as vipers. It further suggests that thermal

72 conditions provide cues for developmental changes in TEWL. Beside plasticity, contrasted

73 individual physiological syndromes could be selected for and contribute to the response to climate

74 change. 
75 Keywords: metabolism, physiology, plasticity, temperature, water loss 


\section{Introduction}

77 Physiological plasticity refers to the ability of an organism to change its physiological state in

78 response to external cues and is an important process for coping with thermal variations (Bonamour

79 et al., 2019; Fuller et al., 2010). In particular, physiological plasticity in response to changes in

80 thermal conditions might determine 'winner or losers' in the context of global warming (Seebacher

81 et al., 2015; Somero, 2010). Thermal plasticity involves a range of distinct but interconnected

82 mechanisms including flexible acclimation responses (shift in physiological routines throughout

83 life) and developmental plasticity (persistent effects induced during development; da Silva et al.,

84 2019; Healy et al., 2019; Sultan, 2017). Recent attention has focused on physiological acclimation

85 capacity to a persistent increase in environmental temperatures (Angilletta, 2009; Huey et al.,

86 2012). Thermal acclimation response for ectotherms involves shifts in physiological responses,

87 which thereby changes the optimal temperature for biological process, and the temperature

88 tolerance limits (Angilletta Jr., 2009; Seebacher, 2005). Such responses are critical to cope with

89 global warming for long-lived ectotherms because of their presumed limited potential for rapid

90 genetic evolution (Chevin et al., 2010; Seebacher et al., 2015; Urban et al., 2014). Yet, uncertainty

91 surrounds the speed and breadth of thermal acclimation responses and their efficiency as a means to

92 buffer ectotherms from global warming (Gunderson \& Stillman, 2015; Havird et al., 2020; Morley

93 et al., 2019; Rohr et al., 2018; Seebacher et al., 2015).

There are several reasons why we might underestimate the true acclimation capacity of long-

95 lived terrestrial ectotherms such as some species of amphibians and reptiles. First, most experiments

96 on these organisms do not last long enough to ensure that individuals are fully acclimated (Rohr et

97 al., 2018; Seebacher et al., 2015). Time to acclimate is typically longer for large-bodied species,

98 based on energetic models and empirical evidence (Kingsolver \& Huey, 2008; Pörtner et al., 2017)

99 and recent comparative analyses across a diversity of ectothermic organisms (Rohr et al., 2018).

100 Long-term (> 1 year) acclimation studies are crucial to investigate the plastic responses of long-

101 lived ectotherm but are very rare. Second, the flexibility of phenotypic responses to acclimation 
102 temperatures is rarely tested for, and how persistent are the effects of developmental plasticity

103 remains unknown for most organisms (Beaman et al., 2016; Ligon et al., 2012). Irreversible

104 acclimation responses to developmental conditions might lead to maladaptive mismatches between

105 the phenotype and ecological conditions in highly variable and unpredictable environments

106 (Gluckman et al., 2007; Piersma and Gils, 2011). Therefore, the ability of organisms to flexibly

107 adjust their phenotype to environmental variations throughout their lifetime may determine their

108 capacity to cope with climate change (Morley et al., 2019; Pallarés et al., 2020). Similarly, whether

109 plastic responses supersede fixed inter-individual differences in physiology has been poorly

110 investigated so far, despite evidence of consistent physiological syndromes in some ectotherms

111 (Goulet et al., 2017a; Mell et al., 2016).

112 In ectotherms, SMR represents the minimal energy requirements to fuel maintenance energy

113 demands, and variation in SMR within and between species may faithfully reflect life history

114 strategies (Clarke, 2006; Seebacher et al., 2015). Considerable variation in MR exists within and

115 between species (Careau et al., 2019), potentially reflecting thermal adaptations (Addo-Bediako et

116 al., 2002) and additive constraints from habitat aridity (Dupoué et al., 2017). Within species,

117 individuals can respond to thermal fluctuations through MR modulation (Noble et al., 2018; While

118 et al., 2018). For instance, after exposure to warm temperatures, MR may decrease ("negative

119 compensation") to limit the energy costs of self-maintenance and minimize oxidative stress (see

120 Costantini, 2008, Angilletta, 2009; Norin et al., 2014). Temperature is also a proximate cue for

121 water constraints inducing adjustment of evaporative water loss (TEWL see Riddell et al., 2019). In

122 terrestrial species, a universal cost of metabolic gas exchange is the loss of water vapor such that

123 there could be a functional trade-off between SMR and TEWL (Woods \& Smith, 2010). Because

124 climate change will affect both energy and water balance, considering jointly SMR, TEWL and

125 associated tradeoffs between both is required (Riddell \& Sears, 2020; Riddell et al., 2018b).

126 Individual variation in metabolism can co-vary with variation in other physiological or in

127 personality traits through consistent pace-of-life syndromes, where "fast" phenotypes are 
128 characterized by a higher SMR (Biro \& Stamps, 2010; Careau et al., 2019, 2014). Thus, phenotypic

129 correlations may also exist between SMR and TEWL and could influence individual sensitivity to

130 temperature and water constraints. Despite its relevance, the long-term developmental effect on

131 variation and co-variation in SMR and TEWL has not been addressed to date, and the pace-of-life

132 syndrome framework has not been extended yet to these functionally related traits.

133 We used a 7-years long experiment to characterize the long-lasting consequences of thermal

134 conditions on the asp viper (Vipera aspis), a temperate ectotherm with a late sexual maturation (age

135 at maturation between 3 to 4 years depending on growth; Bonnet et al., 1999) and a relatively long

136 generation time (maximum lifespan $>10$ years) compared to other squamate reptiles. We

137 hypothesized that thermal conditions should shape both SMR and TEWL, and that chronic exposure

138 would result in persistent effects. We applied three contrasted daily thermal cycles (warm, medium,

139 and cold) with contrasted temperature and thermal amplitude from birth to sexual maturity (4

140 years). Individuals were then placed in a common garden treatment (medium thermal cycle) during

141 their adult life (from 4-7 years old). Building up from previous findings in ectotherms, we tested the

142 following three predictions. First, individuals raised in the warmest thermal cycle should exhibit the

143 lowest SMR due to a negative compensation (Bruton et al., 2012; Clarke, 2006; Zari, 1996).

144 Second, chronic exposure to the thermal treatments during growth should lead to persistent

145 acclimation responses in both SMR and TEWL (Nettle \& Bateson, 2015; Refsnider et al., 2019).

146 Third, SMR should correlate with TEWL among and within individuals because of a functional

147 relationship between the two traits (e.g., water loss caused by ventilation) and/or to some persistent

148 early environment or genetic effects on an underlying trait influencing both SMR and TEWL

149 (Riddell et al., 2018a; Rozen-Rechels et al., 2019).

150

151 Material and Methods

152 Thermal treatment and experimental design 
153 The use of realistic thermal treatment mimicking natural condition is of critical importance to

154 address the impact of climatic changes on physiological responses (Morash et al., 2018; Potter et

155 al., 2013). The asp viper is a diurnal species that behaviorally thermoregulates to reach preferred

156 temperature close to $30^{\circ} \mathrm{C}$ (Lorioux et al., 2013). At night this species will usually hide in a shelter

157 and body temperature will fluctuate with the temperature of the resting habitat. This implies that

158 warmer climate conditions within the thermal critical limits will lead to an extended time spent at

159 the basking temperature, and exposure to warmer nighttime temperatures in vipers (Davy et al.,

160 2017), as predicted by mechanistic models and as seen in empirical studies of heliothermic

161 squamate reptiles (Blouin-Demers \& Weatherhead, 2001). This implies higher mean body

162 temperature and to lower thermal variability in a warmer climate.

163 Therefore, we simulated three realistic daily thermal cycles (Fig. 1A, here after designated

164 as "warm", "medium" and "cold" cycles) that differed in mean temperature $\left(28,24 \& 20^{\circ} \mathrm{C}\right.$

165 respectively) but also in the time at preferred temperature and daily amplitude. Preferred

166 temperature $\left(30^{\circ} \mathrm{C}\right)$ was simulated in all of our treatments but with different durations $(14,9$ and 5

167 hours in warm, medium and cold cycles). We also simulated different night-time temperatures (25,

$16820^{\circ} \mathrm{C}$ and $17^{\circ} \mathrm{C}$ respectively in in warm, medium and cold cycles; see Fig. 1A). Thus, daily thermal

169 amplitude differed between groups $\left(5,10\right.$, and $20^{\circ} \mathrm{C}$ respectively). For all treatments, relative

170 humidity was set at a constant value of $50 \%$ and water vapor pressures deficit were respectively,

$1711.91 \mathrm{kPa}$ (warm cycle), $1.55 \mathrm{kPa}$ (medium cycle) and $1.24 \mathrm{kPa}$ (cold cycle).

We used 49 captive-born snakes from a colony of aspic vipers maintained in the Centre

173 d'Etudes Biologiques de Chizé, France. Mothers were collected in west-central France from

174 neighboring populations and were reproduced in captivity where they were maintained in the same

175 standard conditions during pregnancy (see Lorioux et al., 2013 for details on maintenance

176 conditions and thermal cycles). From birth (September-October 2009) to maturity (October 2013),

177 individuals were randomly assigned within families to one of three treatment $(\mathrm{n}=16,16$ and 17

178 respectively for warm, medium and cold cycle; see Fig. 1B). In November 2013, we transferred all 
179 adult individuals to a common garden at the medium cycle for an additional 3-year period (Fig. 1A).

180 Individuals exposed to the medium cycle during development were thus kept in the same conditions

181 across the experimental timeframe. Individuals from hot and cold cycles were exposed to new

182 thermal conditions when transferred to the common garden, allowing us to test for the flexibility of

183 acclimation responses in these treatment groups.

184 Snakes were housed individually in plastic boxes $(35 \times 25 \times 13 \mathrm{~cm})$ with ad-libitum access

185 to drinking water in a glass bowl. In all experimental treatments, photoperiod followed that of

186 natural day light cycles. We fed the vipers with thawed laboratory mice (Mus musculus). The size of

187 the mice was scaled at $20 \%( \pm 10)$ of the snake mass. Digestion rate, feeding intake and feeding

188 performance increases with temperature in vipers (Naulleau, 1983; Vincent \& Mori, 2007), and the

189 maximum feeding rate is therefore strongly constrained by environmental temperatures. In order to

190 avoid providing food that cannot be processed and digested at low temperatures, we adjusted the

191 feeding regimes to each thermal treatment in order to allow ad libitum feeding rates (every 7, 10

192 and 15 days for warm, medium and cold cycles respectively).

194 Laboratory measurements

195 To investigate how experimental treatments affected growth rate, we weighed $( \pm 0.01 \mathrm{~g})$ and

196 measured (snout-vent-length, $\mathrm{SVL} \pm 0.5 \mathrm{~cm}$ ) all individuals throughout their life. Body mass (BM)

197 was measured at birth and then at the same time points as the respirometry exchanges

198 measurements (see below), while we measured SVL at birth, and when snakes were 30, 49 and 85

199 month-old. To assess both short-term and long-term phenotypic responses, we quantified SMR and

200 TEWL during four successive measurement sessions (Fig. 1B). We measured respiratory exchanges

201 when individuals were 19 and 47 month-old in order to examine to pace of the acclimation response

202 during the development period exposed to contrasted thermal treatments. We conducted

203 measurements 3 months after the beginning of the common garden situation (at 49 month-old) in

204 order to detect any quick changes in SMR or TEWL that may occur following the change in thermal 
205 conditions. Later, we realized a last measurement session 3 years later (at 85 month-old) in order to 206 test for long-term persistent effects of developmental plasticity.

208 Respiratory exchanges

209 We measured the rate of oxygen consumption (VO2 proxy for SMR; ml. $\mathrm{h}^{-1}$ ) and the total

210 evaporative water loss (TEWL; $\left.\mathrm{mg}^{-\mathrm{h}^{-1}}\right)$ on resting individuals at two test temperatures $\left(20^{\circ} \mathrm{C}\right.$ and

$21130^{\circ} \mathrm{C}$ ), to calculate Q10 and acclimation response (Einum et al., 2019; Havird et al., 2020). We

212 randomized temperature trials for each individual. To measure $\mathrm{VO}_{2}$, we used a closed-system

213 respirometry at 19 months post-birth measurements because of the small size of the snakes, and an

214 open-flow respirometry system at 47, 49 and 85 months post-birth measurements (see detailed

215 methods regarding respirometry systems on Appendix S1 in Supporting Information). We measured

216 TEWL together with $\mathrm{VO}_{2}$ using the open flow-system (Fig. 1A). We measured gas exchanges at

217 night on post-absorptive snakes after a minimum of 7 days of fasting. To stabilize the test

218 temperatures and avoid exploratory behaviors, we placed each individual in the temperature-

219 controlled chamber to acclimate for $3 \mathrm{~h}$ prior to each measurement.

221 Statistical analyses

222 All analyses were performed in the R environment, version 3.6.3 (Holding the windsock, (R Core 223 Team, 2020)

225 Linear model to estimate treatment effects on growth rate

226 Growth rate for SVL was computed for each individual during three successive life stages (see

227 Figure 1 and Table 1) and analyzed with a linear model including effects of initial SVL, sex, 228 treatment groups and first order interaction. 
231 We fitted linear mixed models using a Bayesian inference approach with Markov Chain Monte

232 Carlo (MCMC) techniques from the MCMCglmm library (Hadfield, 2009). Results from Bayesian

233 models are further presented as medium estimates $\pm 95 \%$ highest posterior density intervals (HPDI).

234 To investigate independently the effects of experimental conditions on body mass and physiological

235 trait, we first ran univariate mixed effects models with individual identity and mother identity

236 included as random intercepts. In these univariate models, the variance attributed to individual

237 identity represents the intra-individual variance $V_{I}$, whereas the variance attributed to maternal

238 identity represents the intra-litters variance $V_{m}$. For each univariate model, treatment group (warm,

239 medium, or cold cycle), effects of session (see Figure 1), and their interaction were included as

240 fixed factors. The SMR, TEWL, BM trait values were treated as the dependent variable in each

241 univariate model. In univariate models for BM, we included sex as a factor to account for sexual

242 dimorphism. In univariate models for SMR and TEWL, we tested the effect of the ambient

243 temperature during the measurement by including trial temperature $\left(20\right.$ or $\left.30^{\circ} \mathrm{C}\right)$, along with its

244 interactions with treatment and session, as factors. Because BM can strongly influence SMR and

245 TEWL, individual mass at the time of measurement was also included as a covariate in these

246 univariate models but we did not include sex based on preliminary analyses indicating no sexual

247 differences in metabolism and water loss rates.

248 We ran the full model including all variables and interactions following standard guidelines

249 of the MCMCglmm package (Hadfield, 2009). The analysis was performed on raw variables,

250 assuming a Gaussian distribution of the residuals, and poorly informative priors for fixed effects

251 (Gaussian distributed with zero mean and large variance), and uncorrelated poorly informative

252 priors for variance components (inverse Wishart distribution with $n u=0.004$ and $V$ set at 1 ). The

253 MCMC algorithm was set to 120,000 iterations after a minimum burn-in phase of 10,000 iterations

254 to allow convergence and using a thinning interval of 50 to avoid autocorrelation issues. We

255 examined the posterior distribution of model estimates, calculated autocorrelation terms to evaluate

256 independence of the samples in the posterior distributions, and performed half-width tests of 
convergence, which showed satisfactory properties of the sampling distribution. Using the full model, we then tested for the significance of random effects using model comparison with the 259 deviance information criterion (DIC).

261 posterior probability associated to the null hypothesis or pMCMC. To ease interpretation of the 262 results, we further conducted Tukey post-hoc comparisons using the posterior chains of parameter

263 estimates converted into posterior samples of contrasts with the emmeans package (Lenth et al., 264 2020). Note that the results of the MCMCglmm model selection for univariate models were similar 265 to those of classical, maximum likelihood based approaches fitted with the lme procedure (Pinheiro $266 \&$ Bates, 2006).

268 Bivariate mixed model to estimate phenotypic correlation between SMR and TEWL

269 We estimated the phenotypic correlation between SMR and TEWL by including the two as 270 dependent variables in a bivariate mixed model and allowing a heterogeneous residual variance

271 structure. The bivariate model included a regression with body mass, an additive temperature effect, 272 a two-way interaction between treatment and session, residual variance components $V_{e}$ for SMR and 273 TEWL and a covariance between the trait-specific $V_{e}\left(\mathrm{COV}_{e}\right)$, random intercept variance of 274 individual identity $V_{I}$ for SMR and TEWL and a covariance between trait-specific $V_{I}\left(C O V_{I}\right)$. We 275 performed this analysis on centered and scaled variables, assumed a Gaussian distribution of the 276 residuals, and used poorly informative priors for fixed effects (Gaussian distributed with zero mean 277 and large variance) and uncorrelated poorly informative priors for variance components (inverse 278 Wishart distribution with $n u=2$ and $V$ set at 0.5 ). The MCMC algorithm was run for 60,000 279 iterations with a burn-in phase of 10,000 iterations and a thinning interval of 25 . We used the DIC 280 to test the significance of the covariance terms and calculated intra-individual $\left(r_{e}\right)$, inter-individual $281\left(r_{I}\right)$ and total $\left(r_{T}\right)$ phenotypic correlations from the sampled distributions. 
284 We used a $Q_{10}$ formulation of the Arrhenius equation relating metabolic and water loss rates with 285 temperature to calculate the predicted energy budget during inactivity and total evaporative loss 286 during the day for each viper from each treatment at each measurement session. The $Q_{10}$

287 formulation of a process rate $R$ can be written as:

$288 R(T)=R\left(20^{\circ} \mathrm{C}\right) \times Q_{10}^{\frac{(T-20)}{10}}$

289 where $R(T)$ is the predicted process rate at temperature $\mathrm{T}, R\left(20^{\circ} \mathrm{C}\right)$ is our reference process rate at $29020^{\circ} \mathrm{C}$ and $Q_{10}$ is the ratio of the process rate at $30^{\circ} \mathrm{C}$ and $20^{\circ} \mathrm{C}$. We calculated $Q_{10}$ for each viper at 291 each measurement session. We then used equation (1) to calculate the predicted SMR and TEWL 292 over each 30 minutes' time bin from the daily nycthemeral cycle of ambient temperatures for each 293 treatment group of the viper. Daily energy expenditure during inactivity (DEEi; cal.day ${ }^{-1}$ ) and daily 294 total evaporative water loss (DEWL; mg.day ${ }^{-1}$ ) were calculated by summing the predicted SMR and 295 TEWL over each 30-minute time bin through a day. This calculation therefore accounts for both 296 acute effects of temperature (through individual $Q_{10}$ and the nycthemeral cycle) and acclimation 297 effects of temperature (through changes in $\mathrm{R}\left(20^{\circ} \mathrm{C}\right)$ and $Q_{10}$ among treatments. To quantify the 298 degree of metabolic and water loss compensation induced by the observed acclimation response, we 299 compared the DEEi and DEWL of each treatment group at each session with those predicted by a 300 null model. Individuals had lower SMR in response to warmer and less variable temperatures (see 301 results below). Thus, in order to quantify how much individuals saved energy through acclimation 302 to warmer and less variable temperatures, the null model for DEEi assumed no warm acclimation 303 response. We therefore used the mean $R\left(20^{\circ} \mathrm{C}\right)$ and $Q_{10}$ of vipers from the cold cycle to predict the 304 DEEi from the warm and medium cycle treatment in the absence of metabolic acclimation. We 305 estimated the degree of metabolic compensation only at 19 and 47 months post-birth because the 306 acclimation response in SMR did not persist at adulthood in the common garden (49 and 85 months 307 post-birth, see Fig. 2A). In addition, individuals had lower TEWL in both cold and warm cycle 308 treatment than in medium treatment (see results below). In order to quantify the compensation in 
309 DEWL induced by lower TEWL, the null model for DEWL therefore used the mean $R\left(20^{\circ} \mathrm{C}\right)$ and

$310 Q_{10}$ from vipers from the medium cycle treatment as a baseline reference for the two other groups.

\section{Results}

\section{Morphological differences between treatments}

314 There was strong inter-individual variation in body mass but a negligible variation among

315 littermates in the full model (DIC, full model: -929.76, model without $V_{I}:-644.09$, model without

$316 V_{m}$ : -929.57). The change in body mass through time was weakly influenced by a sex-specific effect

317 of treatment groups (DIC, full model: -929.76; full model without three-way interaction: -927.7). In

318 females, body mass did not change between treatments but increased through time faster than in

319 males, especially between session 2 and session 3 (analysis on subset of females data; DIC, model

320 with Treatment $\times$ Time: -453.2 , model without Treatment $\times$ Time: -457.7$)$. In males, body mass

321 was weakly influenced by treatments (analysis on subset of males data; DIC, model with Treatment

$322 \times$ Time: -475.02 , model without Treatment $\times$ Time: -473.98 ) because males from the cold cycle

323 were slightly bigger at 19 months post-birth than males from the two other treatments (Tukey post-

324 hoc contrast between cold and warm cycle: $0.104[-0.034,0.244]$ 95\% HPDI, warm and medium

325 cycle: 0.099 [-0.034, 0.237] 95\% HPDI see Table 1). After accounting for these fixed effects, the

326 inter-individual variation in body mass was highly significant $\left(R_{I}=0.660[0.523,0.743] 95 \%\right.$

327 HPDI).

328

329 Growth rate measured in each life stage differed between sexes consistently (faster growth in

330 females, all $\mathrm{P}<0.001)$ and also between treatments from birth to 30 months post-birth (

$\left.331 F_{2,44}=6.69, P=0.003\right)$ and from 49 months post-birth to 85 months post-birth $\left(F_{2,44}=3.75, P=0.03\right.$

332 ). During the acclimation phase, vipers grew longer in the warm cycle than in the medium and cold

333 cycle. During the common garden phase at adulthood, there was a compensatory growth response in 
334 vipers from the medium and cold cycle, which reached slightly higher SVL than vipers from the

335 warm cycle group at 85 months post-birth (see Table 1).

337 Univariate models for SMR and TEWL

338 According to our model selection procedure, there was strong inter-individual variation in SMR and

339 comparatively little variation among littermates in the full model (DIC, full model: -186.69 , model

340 without $V_{I}:-171.7$, model without $V_{m}:-186.03$ ). Regarding the fixed effects (see Table S2), SMR

341 increased with body mass (pMCMC $>0.001)$ and was higher at $30^{\circ} \mathrm{C}$ than at $20^{\circ} \mathrm{C}(\mathrm{pMCMC}>$

342 0.001), but neither the treatment group nor the trial temperature influenced the allometric

343 relationship with body mass ( $\mathrm{pMCMC}>0.10)$. Independently from body mass, treatment groups

344 influenced SMR differently through time and trial temperature levels (DIC, model without $V_{m}$ and

345 without Treatment $\times$ Time $\times$ Temperature: -182.3 ). At $20^{\circ} \mathrm{C}$, vipers raised in the warm cycle

346 treatment had slightly lower SMR than vipers raised in the medium and cold cycles during the

347 acclimation phase (19 and 47 months post-birth) and this difference was significant three months

348 after the end of the manipulation (49 months post-birth; post-hoc contrasts of the median posterior

349 distribution, see Fig. 2A). Two years later, at 85 months post-birth, SMR values were not different

350 between treatments irrespective of body temperatures. At $30^{\circ} \mathrm{C}$, vipers raised in the warm cycle

351 treatment had significantly lower SMR than vipers raised in the medium and cold cycles during the 352 acclimation phase (19 and 47 months post-birth), but this difference vanished right after the end of

353 the manipulation (49 and 85 months post-birth, see Fig. 2A). After accounting for these fixed

354 effects, the inter-individual variation in SMR was significant but relatively small $\left(R_{I}=0.129[0.049\right.$,

$3550.231] 95 \%$ HPDI).

357 Similarly, there was inter-individual variation in TEWL and comparatively little variation among

358 littermates in the full model (DIC, full model: -97.7, model without $V_{I}:-66.2$, model without $V_{m}$ : -

359 97.9). The TEWL increased with body mass similarly between treatment and temperature groups 
$(\mathrm{pMCMC}>0.05)$ and was higher at $30^{\circ} \mathrm{C}$ than at $20^{\circ} \mathrm{C}(\mathrm{pMCMC}>0.001)$. Contrary to SMR,

361 treatment groups significantly influenced TEWL independently from time and trial temperature (see

362 Table S2). On average, vipers raised in the medium cycle treatment displayed consistently higher

363 TEWL rates through lifetime relative to the warm treatment (Tukey post-hoc contrast: 0.1203

$364[0.0281,0.2141] 95 \%$ HPDI) and also slightly higher TEWL than in the cold cycle treatment

365 (Tukey post-hoc contrast: 0.092 [-0.0035, 0.1827] 95\% HPDI) irrespective of trial temperature (see

366 Fig. 2B). After accounting for these fixed effects, the inter-individual variation in TEWL was

367 significant and twice than the one for $\operatorname{SMR}\left(R_{I}=0.237[0.115,0.373] 95 \%\right.$ HPDI $)$.

369 Bivariate models for SMR and TEWL

370 The bivariate mixed models indicated that both the intra-individual and inter-individual covariance

371 between SMR and TEWL were positive and significantly different from zero as shown by the DIC

372 (full model: $\mathrm{DIC}=1173.91, C O V_{e}$ set to zero: $\mathrm{DIC}=1268.6 ; C O V_{I}$ set to zero: $\mathrm{DIC}=1198.21$; see

373 Table 2). The inter-individual correlation was slightly larger $\left(r_{I}=0.69[0.46,0.88] 95 \% \mathrm{CI}\right)$ than the

374 intra-individual correlation $\left(r_{e}=0.60[0.51,0.68] 95 \% \mathrm{CI}\right)$ and the total phenotypic correlation $\left(r_{P}=\right.$ $3750.44[0.15,0.75]$ 95\%CI, see Fig. 3A and 3B).

377 Metabolic and water loss compensation

378 Individuals from warm and medium cycle treatment groups had lower predicted DEEi than 379 expected in the null model without an acclimation response. Individuals from the warm cycle 380 treatment compensated for approximately $40 \%$ of their DEEi at both 19 and 45 months post-birth.

381 In the medium cycle treatment group, individuals compensated for $28.5 \%$ of their DEEi at 45

382 months post-birth (Table 3, Fig. 4A). Similarly, DEWL was lower than expected in the null model

383 without an acclimation response for individuals from the warm and cold cycle treatment groups. At

38447 months post-birth, DEWL was reduced by $36.4 \%$ for individuals acclimated to the warm cycle 385 treatment, and by $24.2 \%$ for individuals acclimated to the cold cycle treatment. At 49 months post- 
386 birth individuals acclimated to the cold cycle treatment compensated for $32.7 \%$ of their DEWL,

387 and individuals acclimated to the warm cycle treatment compensated for $49.5 \%$ of their DEWL at

38885 months post-birth (Table 3, Fig. 4B).

\section{Discussion}

391 Thermal conditions simulated in three different daily thermal cycles led to developmental changes

392 in TEWL that persisted at adulthood but individuals flexibly adjusted their SMR to thermal

393 conditions experienced in situ. Further, we found that inter-individual variation of these two

394 functional traits was consistent and positively correlated throughout life. This persistent phenotypic

395 (co)variation at the individual level likely reflects a physiological syndrome linking metabolism and 396 water loss.

398 Negative compensation and flexible acclimation in SMR

399 During the time period of exposure to contrasted treatments (until the age of 4 years old), snakes

400 exposed to the warmest and less variable thermal conditions adjusted their SMR with a negative

401 compensatory response (Clarke, 2006; Dupoué et al., 2017) when compared to snakes maintained at

402 lower and more variable temperatures (medium and cold cycle). This physiological response to

403 thermal conditions did not persist in a common garden, providing unambiguous evidence that

404 acclimation in SMR was flexible. The compensatory response was quantified and allowed vipers to

405 save as much as $40 \%$ of their DEEi relative to a null model hypothetical estimate simulating no

406 acclimation response. The asp viper is a typical capital breeder with a low feeding frequency and a

407 slow pace of life strategy. Therefore, lower SMR is likely to be advantageous according to the

408 "allocation model" of energy metabolism where individuals with lower SMR benefit from more

409 energy to spend in maintenance (e.g., survival), activity and/or reproduction (Careau et al., 2008;

410 Mathot \& Dingemanse, 2015). In support of this scenario, we found that warm-acclimated vipers

411 had both the lowest SMR and the highest growth rate. Thus, warm-acclimated snakes were able to 
412 advantageously invest more energy into growth, given their lower energetic demands. Even though

413 the relationship between SMR and fitness can be quite variable across organisms (Arnold et al.,

414 2021), these results confirm earlier findings where MR reduction in warm-acclimated ectotherms

415 minimized the daily energetic costs of self-maintenance and limited oxidative stress by decreasing

416 respiratory activity (Norin et al., 2014; Seebacher et al., 2010). In addition, the covariation between

417 SMR and TEWL suggests that lower SMR is likely to positively impact water balance through

418 reduced respiratory water loss (Dupoué et al., 2015). In colder conditions, MR may remain high to

419 support performance, activity, and physiological functions despite lower temperatures (Berg et al.,

420 2017). We further found that treatment-induced changes in SMR differed at body temperatures of

42120 and $30^{\circ} \mathrm{C}$, suggesting that thermal acclimation changed also the acute effects of temperature on

422 metabolism (Xie et al., 2017).

423 Both the mean temperature and the thermal variability from each daily cycle could drive

424 these acclimation responses (Kern et al., 2015). At a given mean temperature, it has been suggested

425 that a higher daily thermal fluctuation can lead to an increased tolerance to extreme temperature

426 (Verheyen \& Stoks, 2019). However, the effects of temporal variation of temperatures on

427 acclimation responses of metabolism and other functional processes are often species dependent in

428 ectotherms (Niehaus et al., 2011; Noer et al., 2020), and can also interact with changes in mean

429 temperature (Bozinovic et al., 2011; Colinet et al., 2015). Further studies are required to elucidate

430 whether acclimation responses of SMR and other functional traits in asp vipers are causally linked

431 with changes in mean temperature, changes in the thermal variability or both. Our results however

432 likely illustrate more realistic responses to climate change compared to findings from studies

433 focusing on acclimation responses at a constant daily temperature because animals are exposed to

434 climate variability in their environment and predictions from laboratory studies in complex thermal

435 environments are more likely to predict future changes in natural populations (Morash et al., 2018).

436 Some SMR differences persisted among groups for a month after the beginning of the

437 common garden ( 49 months post-birth, at $20^{\circ} \mathrm{C}$ ), but no group difference in SMR was found 3 years 
438 later (85 months post-birth). This indicates that SMR responds dynamically to the thermal

439 environment to optimize performance and satisfy daily energetic costs (Berg et al., 2017). Such

440 reversibility is adaptive in seasonal, variable, or unpredictable environments (Piersma \& Gils,

441 2011), and thus was likely selected for in temperate ectotherms such as the aspic viper (Little \&

442 Seebacher, 2016). In turn, the inherent capacity of these organisms to reversibly shift their SMR in

443 response to fluctuating temperatures significantly buffers their energetic sensitivity to climate

444 change and to extreme weather events by allowing them to compensate for the energy costs of life

445 at warmer temperatures (Pallarés et al., 2020; Seebacher et al., 2015). Flexible metabolic shifts may

446 be achieved by a range of short-term proximal mechanisms, such as changes in the structure

447 (membrane fluidity, protein activity) and density of mitochondria (Chung et al., 2017; Norin \&

448 Metcalfe, 2019; Seebacher et al., 2010). Exploring the mechanisms underlying thermal acclimation

449 of SMR in vipers would require further functional studies of their energy metabolism, especially

450 detailed analyses of the thermal plasticity of mitochondrial activity. Additional experimental design

451 could also help to fully disentangle between developmental plasticity and thermal acclimation sensu

452 stricto by investigating how flexible is SMR plasticity to temperature at different stages through life

453 (Beaman et al., 2016).

454

455 Developmental plasticity in TEWL

456 Thermal conditions during development induced long-lasting effects on TEWL across the

457 experimental timeframe. Thus, our study provides a clear demonstration that, at a given relative

458 humidity, mean temperature and thermal variability can lead to developmental plasticity in a key

459 functional trait related to water balance. Individuals raised in both the warmest and the coldest

460 conditions (warm and cold cycles) had lower TEWL rates on average than those raised in the

461 intermediate conditions (medium cycle treatment). Metabolic compensation associated with the

462 warm cycle treatment may have contributed to the decrease in TEWL that we observed in this group

463 as lower metabolic gas exchanges are associated with reduced loss of water vapor (Dupoué et al., 
464 2015; Riddell et al., 2018a). However, individuals raised in the cold treatment also had low

465 evaporative water loss, despite their high oxygen consumption rates. We calculated the daily

466 evaporative water loss (DEWL) taking into account solely the thermal dependence of TEWL. This

467 showed that individuals from warm and cold cycles were able to reduce their DEWL by

468 approximatively $30 \%$, and even up to $50 \%$ for individuals from the warm cycle treatment group at

46985 months post-birth. The decrease of TEWL for snakes from the warm cycle treatment may thus

470 be adaptive in anticipation of hotter and drier conditions by reducing DEWL and thus reducing the

471 risks of dehydration. The fact that TEWL also decreased under the cold cycle treatment remains

472 intriguing because the water vapor deficit was minimal and vipers had low predicted DEWL in this

473 treatment group. This reduction in cold conditions could reflect a plastic response of TEWL to the

474 lower absolute air humidity in this treatment or to some behavioral constraints (less frequent

475 drinking behavior) imposed by activity restriction in cold environments. Our results suggest that

476 mean and variance of temperature can provide a relevant cue to regulate water balance (Riddell et

477 al., 2019) probably because temperature, water vapor pressure deficit and water constraints are

478 correlated in natural habitats and organisms cue on temperature to assess dehydration risks (Dai,

479 2013). Manipulating both temperature and humidity levels will be required to elucidate how

480 temperature and humidity levels jointly influence TEWL plasticity (Rozen-Rechels et al., 2019).

481 Heretofore, the pace of TEWL plasticity and the period of sensitivity during development also

482 remain unknown, as well as the proximal mechanisms involved.

484 Persistent individual differences and covariation between SMR and TEWL

485 Phenotypic (Baškiera \& Gvoždík, 2020; Careau et al., 2019; Nilsson et al., 2009) and quantitative 486 genetic (Pettersen et al., 2018) studies of energy metabolism have uncovered that MR and its 487 thermal sensitivity are often consistent over time and variable among individuals within the same 488 species (Nespolo et al., 2003; Nespolo \& Franco, 2007; Réveillon et al., 2019). In line with these 489 results, our study shows consistent inter-individual variation in SMR throughout 7 years of life and, 
490 for the first time, also in TEWL. In addition, SMR and TEWL were strongly positively correlated

491 both within and among individuals and so independently of developmental treatments. The quite

492 remarkable age span over which we examined these individual differences suggests very strong and

493 persistent effects of pre-natal factors on these two functional traits regardless of post-natal

494 conditions and growth trajectories. Our dataset does not allow to elucidate the origin of these

495 physiological syndromes that possibly include long-lasting effects of early environmental

496 conditions and/or genetic factors (White et al., 2019).

497 The pace-of-life syndrome describes covariation between MR and individual personality,

498 behavior, and life history traits. It is an useful framework to understand how variation in SMR may

499 correlate with maximum MR, behavioral activity and risk-prove behaviors (Careau et al., 2019,

500 2014; Mell et al., 2016). This theoretical framework has recently been extended to thermal

501 physiology where inter-individual in MR and behavior coincide with variation along the cold-hot

502 axis of thermal physiology (Goulet et al., 2017a; Goulet et al., 2017b). Our results suggest that such

503 a physiological syndrome can also integrate functional traits related to water and energy balance.

504 Although this is the first quantitative analysis of this physiological syndrome, the shared proximal

505 mechanisms, joint plasticity and potential co-evolution between functional traits related to energy

506 balance, respiration, thermal physiology and water biology have already been emphasized (Riddell

507 et al., 2019, 2018a; Rozen-Rechels et al., 2019).

508

509 Conclusion

510 Vipers exhibited two distinct plastic responses to thermal treatment: a phenotypic flexibility of

511 SMR and persistent developmental plasticity in TEWL. In addition, SMR and TEWL strongly co-

512 varied both within and among individuals over their lifespan. Therefore, terrestrial ectotherms can

513 simultaneously exhibit strong individual variation, reflecting physiological syndromes and likely

514 genetic variation, and plastic responses in two functional traits related to thermal and water balance.

515 These two sources of variability may allow these organisms to cope with climate change by 
516 combining plastic, adaptive responses to droughts and heatwaves (Gunderson et al., 2017;

517 Seebacher et al., 2015) and responses to selection on physiological syndromes (Žagar et al., 2018).

518

519 References

520 Addo-Bediako, A., Chown, S.L., Gaston, K.J., 2002. Metabolic Cold Adaptation in Insects: A

$521 \quad$ Large-Scale Perspective. Funct. Ecol. https://doi.org/10.2307/826586

522 Angilletta Jr., M.J., 2009. Thermal Adaptation. Oxford University Press.

$523 \quad$ https://doi.org/10.1093/acprof:oso/9780198570875.001.1

524 Arnold, P.A., Delean, S., Cassey, P., White, C.R., 2021. Meta-analysis reveals that resting

525 metabolic rate is not consistently related to fitness and performance in animals. J. Comp.

526 Physiol. B 1-14. https://doi.org/10.1007/s00360-021-01358-w

527 Baškiera, S., Gvoždík, L., 2020. Repeatability and heritability of resting metabolic rate in a long-

528 lived amphibian. Comp. Biochem. Physiol. Part A Mol. Integr. Physiol. 253, 110858.

529 https://doi.org/10.1016/j.cbpa.2020.110858

530 Beaman, J.E., White, C.R., Seebacher, F., 2016. Evolution of Plasticity: Mechanistic Link between

531 Development and Reversible Acclimation. Trends Ecol. Evol.

532 https://doi.org/10.1016/j.tree.2016.01.004

533 Berg, W., Theisinger, O., Dausmann, K.H., 2017. Acclimatization patterns in tropical reptiles:

534 uncoupling temperature and energetics. Sci. Nat. https://doi.org/10.1007/s00114-017-1506-0

535 Biro, P.A., Stamps, J.A., 2010. Do consistent individual differences in metabolic rate promote

536 consistent individual differences in behavior? Trends Ecol. Evol.

537 https://doi.org/10.1016/j.tree.2010.08.003

538 Blouin-Demers, G., Weatherhead, P.J., 2001. Thermal ecology of black rat snakes (Elaphe obsoleta)

539 in a thermally challenging environment. Ecology 82, 3025-3043. https://doi.org/10.1890/0012-

$540 \quad 9658(2001) 082[3025: T E O B R S] 2.0 . C O ; 2$

541 Bonamour, S., Chevin, L.M., Charmantier, A., Teplitsky, C., 2019. Phenotypic plasticity in 
response to climate change: The importance of cue variation. Philos. Trans. R. Soc. B Biol.

\section{$543 \quad$ Sci. https://doi.org/10.1098/rstb.2018.0178}

544 Bonnet, X., Naulleau, G., Lourdais, O., 1999. Growth in the asp viper (Vipera aspis L.): insights 545 from long term field study. Curr. Stud. Herpetol.

546 Bozinovic, F., Bastías, D.A., Boher, F., Clavijo-Baquet, S., Estay, S.A., Angilletta, M.J., 2011. The 547 mean and variance of environmental temperature interact to determine physiological tolerance 548 and fitness. Physiol. Biochem. Zool. 84, 543-552. https://doi.org/10.1086/662551

549 Bruton, M.J., Cramp, R.L., Franklin, C.E., 2012. Benefits of thermal acclimation in a tropical 550 aquatic ectotherm, the Arafura filesnake, Acrochordus arafurae. J. Comp. Physiol. B Biochem. 551 Syst. Environ. Physiol. 182, 541-551. https://doi.org/10.1007/s00360-011-0643-6

552 Careau, V., Beauchamp, P.P., Bouchard, S., Morand-Ferron, J., 2019. Energy metabolism and 553 personality in wild-caught fall field crickets. Physiol. Behav.

$554 \quad$ https://doi.org/10.1016/j.physbeh.2018.11.023

555 Careau, V., Gifford, M.E., Biro, P.A., 2014. Individual (co)variation in thermal reaction norms of 556 standard and maximal metabolic rates in wild-caught slimy salamanders. Funct. Ecol. $557 \quad$ https://doi.org/10.1111/1365-2435.12259

558 Careau, V., Thomas, D., Humphries, M.M., Réale, D., 2008. Energy metabolism and animal 559 personality. Oikos. https://doi.org/10.1111/j.0030-1299.2008.16513.x

560 Chevin, L.-M., Lande, R., Mace, G.M., 2010. Adaptation, Plasticity, and Extinction in a Changing 561 Environment: Towards a Predictive Theory. PLoS Biol. 8, e1000357. 562 https://doi.org/10.1371/journal.pbio.1000357

563 Chung, D.J., Bryant, H.J., Schulte, P.M., 2017. Thermal acclimation and subspecies-specific effects 564 on heart and brain mitochondrial performance in a eurythermal teleost (Fundulus heteroclitus). 565 J. Exp. Biol. 220, 1459-1471. https://doi.org/10.1242/jeb.151217

566 Clarke, A., 2006. Temperature and the metabolic theory of ecology. Funct. Ecol. 20, 405-412. 567 https://doi.org/10.1111/j.1365-2435.2006.01109.x 
568 Colinet, H., Sinclair, B.J., Vernon, P., Renault, D., 2015. Insects in Fluctuating Thermal

569 Environments. Annu. Rev. Entomol. 60, 123-140. https://doi.org/10.1146/annurev-ento-

$570 \quad 010814-021017$

571 Costantini, D., 2008. Oxidative stress in ecology and evolution: lessons from avian studies. Ecol.

572 Lett. 11, 1238-1251. https://doi.org/10.1111/j.1461-0248.2008.01246.x

573 da Silva, C.R.B., Riginos, C., Wilson, R.S., 2019. An intertidal fish shows thermal acclimation

574 despite living in a rapidly fluctuating environment. J. Comp. Physiol. B 20191893 189, 385-

575 398. https://doi.org/10.1007/S00360-019-01212-0

576 Dai, A., 2013. Increasing drought under global warming in observations and models. Nat. Clim.

577 Chang. 3, 52-58. https://doi.org/10.1038/nclimate1633

578 Davy, R., Esau, I., Chernokulsky, A., Outten, S., Zilitinkevich, S., 2017. Diurnal asymmetry to the 579 observed global warming. Int. J. Climatol. 37, 79-93. https://doi.org/10.1002/joc.4688

580 Dupoué, A., Brischoux, F., Lourdais, O., 2017. Climate and foraging mode explain interspecific 581 variation in snake metabolic rates. Proc. R. Soc. B Biol. Sci. 284.

582 https://doi.org/10.1098/rspb.2017.2108

583 Dupoué, A., Stahlschmidt, Z.R., Michaud, B., Lourdais, O., 2015. Physiological state influences 584 evaporative water loss and microclimate preference in the snake Vipera aspis. Physiol. Behav. $585 \quad$ https://doi.org/10.1016/j.physbeh.2015.02.042

586 Einum, S., Ratikainen, I., Wright, J., Pélabon, C., Bech, C., Jutfelt, F., Stawski, C., Burton, T., 587 2019. How to quantify thermal acclimation capacity? Glob. Chang. Biol. 25, 1893-1894. $588 \quad$ https://doi.org/10.1111/gcb.14598

589 Fuller, A., Dawson, T.J., Helmuth, B., Hetem, R.S., Mitchell, D., Maloney, S.K., 2010.

590 Physiological mechanisms in coping with climate change. Physiol. Biochem. Zool. 83, 713$591 \quad 720$.

592 Gluckman, P.D., Hanson, M.A., Beedle, A.S., 2007. Early life events and their consequences for 593 later disease: A life history and evolutionary perspective. Am. J. Hum. Biol. 19, 1-19. 
595 Goulet, C.T., Thompson, M.B., Chapple, D.G., 2017a. Repeatability and correlation of 596 physiological traits: Do ectotherms have a "thermal type"? Ecol. Evol. 7, 710-719. https://doi.org/10.1002/ece3.2632

598 Goulet, C.T., Thompson, M.B., Michelangeli, M., Wong, B.B.M., Chapple, D.G., 2017b. Thermal 599 physiology: A new dimension of the pace-of-life syndrome. J. Anim. Ecol. 86, 1269-1280. $600 \quad$ https://doi.org/10.1111/1365-2656.12718

601 Gunderson, A.R., Dillon, M.E., Stillman, J.H., 2017. Estimating the benefits of plasticity in 602 ectotherm heat tolerance under natural thermal variability. Funct. Ecol. $603 \quad$ https://doi.org/10.1111/1365-2435.12874

604 Gunderson, A.R., Stillman, J.H., 2015. Plasticity in thermal tolerance has limited potential to buffer 605 ectotherms from global warming. Proc. R. Soc. B Biol. Sci.

606 https://doi.org/10.1098/rspb.2015.0401

607 Hadfield, J., 2009. MCMC Methods for Multi-response Generalized Linear Mixed Models. The 608 MCMCglmm R Package.

609 Havird, J.C., Neuwald, J.L., Shah, A.A., Mauro, A., Marshall, C.A., Ghalambor, C.K., 2020.

610 Distinguishing between active plasticity due to thermal acclimation and passive plasticity due 611 to Q10 effects: Why methodology matters. Funct. Ecol. 34, 1015-1028. https://doi.org/10.1111/1365-2435.13534

613 Healy, T.M., Bock, A.K., Burton, R.S., 2019. Variation in developmental temperature alters 614 adulthood plasticity of thermal tolerance in Tigriopus californicus. J. Exp. Biol. 222. 615 https://doi.org/10.1242/JEB.213405

616 Huey, R.B., Kearney, M.R., Krockenberger, A., Holtum, J.A.M., Jess, M., Williams, S.E., 2012. 617 Predicting organismal vulnerability to climate warming: Roles of behaviour, physiology and 618 adaptation. Philos. Trans. R. Soc. B Biol. Sci. 367, 1665-1679.

619 https://doi.org/10.1098/rstb.2012.0005 
620 Kern, P., Cramp, R.L., Franklin, C.E., 2015. Physiological responses of ectotherms to daily temperature variation. J. Exp. Biol. 218, 3068-3076. https://doi.org/10.1242/jeb.123166

622 Kingsolver, J.G., Huey, R.B., 2008. Size, temperature, and fitness: three rules, Evolutionary 623 Ecology Research. Evolutionary Ecology, Ltd.

624 Lenth, R., Singmann, H., Love, J., Buerkner, P., Herve, M., 2020. emmeans: estimated marginal 625 means. R package version 1.4.4. Am. Stat 34, 216-221.

626 Ligon, D.B., Peterson, C.C., Lovern, M.B., 2012. Acute and Persistent Effects of Pre- and 627 Posthatching Thermal Environments on Growth and Metabolism in the Red-Eared Slider 628 Turtle, Trachemys scripta elegans. J. Exp. Zool. Part A Ecol. Genet. Physiol. 317, 227-235. 629 https://doi.org/10.1002/jez.1716

630 Little, A., Seebacher, F., 2016. Acclimation, acclimatization, and seasonal variation in amphibians and reptiles, in: Amphibian and Reptile Adaptations to the Environment: Interplay between Physiology and Behavior. pp. 41-62.

633 Lorioux, S., Vaugoyeau, M., Denardo, D.F., Clobert, J., Guillon, M., Lourdais, O., 2013. Stage 634 dependence of phenotypical and phenological maternal effects: insight into squamate reptile reproductive strategies. Am. Nat. 182, 223-33. https://doi.org/10.1086/670809

641 Morash, A.J., Neufeld, C., MacCormack, T.J., Currie, S., 2018. The importance of incorporating 642 natural thermal variation when evaluating physiological performance in wild species. J. Exp. 643 Biol. https://doi.org/10.1242/jeb.164673

644 Morley, S.A., Peck, L.S., Sunday, J.M., Heiser, S., Bates, A.E., 2019. Physiological acclimation and 645 persistence of ectothermic species under extreme heat events. Glob. Ecol. Biogeogr. 28, 1018- 
647 Naulleau, G., 1983. The Effects of Temperature on Digestion in Vipera aspis. J. Herpetol. 17, 166. $648 \quad$ https://doi.org/10.2307/1563457

649 Nespolo, R.F., Franco, M., 2007. Whole-animal metabolic rate is a repeatable trait: A meta650 analysis. J. Exp. Biol. 210, 2000-2005. https://doi.org/10.1242/jeb.02780

651 Nespolo, R.F., Lardies, M.A., Bozinovic, F., 2003. Intrapopulational variation in the standard 652 metabolic rate of insects: Repeatability, thermal dependence and sensitivity (Q10) of oxygen consumption in a cricket. J. Exp. Biol. 206, 4309-4315. https://doi.org/10.1242/jeb.00687

654 Nettle, D., Bateson, M., 2015. Adaptive developmental plasticity: What is it, how can we recognize 655 it and when can it evolve? Proc. R. Soc. B Biol. Sci. 282.

656 https://doi.org/10.1098/rspb.2015.1005

657 Niehaus, A.C., Wilson, R.S., Seebacher, F., Franklin, C.E., 2011. Striped marsh frog 658 (limnodynastes peronii) tadpoles do not acclimate metabolic performance to thermal 659 variability. J. Exp. Biol. 214, 1965-1970. https://doi.org/10.1242/jeb.054478

660 Nilsson, J.-Å., Åkesson, M., Nilsson, J.F., 2009. Heritability of resting metabolic rate in a wild 661 population of blue tits. J. Evol. Biol. 22, 1867-1874. https://doi.org/10.1111/j.1420$6629101.2009 .01798 . x$

663 Noble, D.W.A., Stenhouse, V., Schwanz, L.E., 2018. Developmental temperatures and phenotypic 664 plasticity in reptiles: a systematic review and meta-analysis. Biol. Rev. 93, 72-97. 665 https://doi.org/10.1111/brv.12333

666 Noer, N.K., Pagter, M., Bahrndorff, S., Malmendal, A., Kristensen, T.N., 2020. Impacts of thermal 667 fluctuations on heat tolerance and its metabolomic basis in Arabidopsis thaliana, Drosophila 668 melanogaster, and Orchesella cincta. PLoS One 15, e0237201.

669 https://doi.org/10.1371/journal.pone.0237201

670 Norin, T., Malte, H., Clark, T.D., 2014. Aerobic scope does not predict the performance of a 671 tropical eurythermal fish at elevated temperatures. J. Exp. Biol. 217, 244-251. 
673 Norin, T., Metcalfe, N.B., 2019. Ecological and evolutionary consequences of metabolic rate 674 plasticity in response to environmental change. Philos. Trans. R. Soc. B Biol. Sci. 675 https://doi.org/10.1098/rstb.2018.0180

676 Pallarés, S., Colado, R., Botella-Cruz, M., Montes, A., Balart-García, P., Bilton, D.T., Millán, A., 677 Ribera, I., Sánchez-Fernández, D., 2020. Loss of heat acclimation capacity could leave 678 subterranean specialists highly sensitive to climate change. Anim. Conserv. 1-9. 679 https://doi.org/10.1111/acv.12654

680 Pettersen, A.K., Marshall, D.J., White, C.R., 2018. Understanding variation in metabolic rate. J. $681 \quad$ Exp. Biol. https://doi.org/10.1242/jeb.166876

682 Piersma, T., Gils, J.A. van., 2011. The flexible phenotype: a body-centred integration of ecology, 683 physiology, and behaviour. Oxford University Press.

684 Pinheiro, J., Bates, D., 2006. Mixed-Effects Models in S and S-PLUS.

685 Pörtner, H.O., Bock, C., Mark, F.C., 2017. Oxygen- \& capacity-limited thermal tolerance: Bridging 686 ecology \& physiology. J. Exp. Biol. https://doi.org/10.1242/jeb.134585

687 Potter, K.A., Woods, H.A., Pincebourde, S., 2013. Microclimatic challenges in global change 688 biology. Glob. Chang. Biol. 19, 2932-2939. https://doi.org/10.1111/GCB.12257

689 R Core Team, 2020. R: A Language and Environment for Statistical Computing. 690 pdfs.semanticscholar.org.

691 Refsnider, J.M., Clifton, I.T., Vazquez, T.K., 2019. Developmental plasticity of thermal ecology 692 traits in reptiles: Trends, potential benefits, and research needs. J. Therm. Biol. 693 https://doi.org/10.1016/j.jtherbio.2019.06.005

694 Réveillon, T., Rota, T., Chauvet, É., Lecerf, A., Sentis, A., 2019. Repeatable inter-individual 695 variation in the thermal sensitivity of metabolic rate. Oikos 128, 1633-1640. 696 https://doi.org/10.1111/oik.06392

697 Riddell, E.A., McPhail, J., Damm, J.D., Sears, M.W., 2018a. Trade-offs between water loss and gas 
exchange influence habitat suitability of a woodland salamander. Funct. Ecol. 32, 916-925. https://doi.org/10.1111/1365-2435.13030

700

701

702

703 704

705

706

707

708

709

710

711

712 713

Riddell, E.A., Odom, J.P., Damm, J.D., Sears, M.W., 2018b. Plasticity reveals hidden resistance to extinction under climate change in the global hotspot of salamander diversity. Sci. Adv. 4. https://doi.org/10.1126/sciadv.aar5471

Riddell, E.A., Roback, E.Y., Wells, C.E., Zamudio, K.R., Sears, M.W., 2019. Thermal cues drive plasticity of desiccation resistance in montane salamanders with implications for climate change. Nat. Commun. 10, 4091. https://doi.org/10.1038/s41467-019-11990-4

Riddell, E.A., Sears, M.W., 2020. Terrestrial Salamanders Maintain Habitat Suitability under Climate Change despite Trade-Offs between Water Loss and Gas Exchange. Physiol. Biochem. Zool. https://doi.org/10.1086/709558

Rohr, J.R., Civitello, D.J., Cohen, J.M., Roznik, E.A., Sinervo, B., Dell, A.I., 2018. The complex drivers of thermal acclimation and breadth in ectotherms. Ecol. Lett. 21, 1425-1439. https://doi.org/10.1111/ele.13107

Rozen-Rechels, D., Dupoué, A., Lourdais, O., Chamaillé-Jammes, S., Meylan, S., Clobert, J., Le Galliard, J., 2019. When water interacts with temperature: Ecological and evolutionary implications of thermo-hydroregulation in terrestrial ectotherms. Ecol. Evol. 9, 10029-10043. https://doi.org/10.1002/ece3.5440

Seebacher, F., 2005. A review of thermoregulation and physiological performance in reptiles: What is the role of phenotypic flexibility? J. Comp. Physiol. B Biochem. Syst. Environ. Physiol. https://doi.org/10.1007/s00360-005-0010-6

Seebacher, F., Brand, M.D., Else, P.L., Guderley, H., Hulbert, A.J., Moyes, C.D., 2010. Plasticity of oxidative metabolism in variable climates: molecular mechanisms. Physiol. Biochem. Zool. 83, 721-32. https://doi.org/10.1086/649964

Seebacher, F., White, C.R., Franklin, C.E., 2015. Physiological plasticity increases resilience of ectothermic animals to climate change. Nat. Clim. Chang. 
725 Somero, G.N., 2010. The physiology of climate change: How potentials for acclimatization and genetic adaptation will determine "winners" and "losers." J. Exp. Biol. 213, 912-920. https://doi.org/10.1242/jeb.037473

728 Sultan, S.E., 2017. Developmental plasticity: Re-conceiving the genotype. Interface Focus.

729 https://doi.org/10.1098/rsfs.2017.0009

730 Urban, M.C., Richardson, J.L., Freidenfelds, N.A., 2014. Plasticity and genetic adaptation mediate

731 amphibian and reptile responses to climate change. Evol. Appl. https://doi.org/10.1111/eva.12114

733 Verheyen, J., Stoks, R., 2019. Temperature variation makes an ectotherm more sensitive to global

734 warming unless thermal evolution occurs. J. Anim. Ecol. 88, 624-636.

$735 \quad$ https://doi.org/10.1111/1365-2656.12946

736 Vincent, S.E., Mori, A., 2007. Determinants of feeding performance in free-ranging pit-vipers

737 (Viperidae: Ovophis okinavensis): key roles for head size and body temperature. Biol. J. Linn.

738 Soc. 93, 53-62. https://doi.org/10.1111/j.1095-8312.2007.00928.x

739 While, G.M., Noble, D.W.A., Uller, T., Warner, D.A., Riley, J.L., Du, W.-G., Schwanz, L.E., 2018.

740 Patterns of developmental plasticity in response to incubation temperature in reptiles. J. Exp.

741 Zool. Part A Ecol. Integr. Physiol. 329, 162-176. https://doi.org/10.1002/jez.2181

742 White, C.R., Marshall, D.J., Alton, L.A., Arnold, P.A., Beaman, J.E., Bywater, C.L., Condon, C.,

743 Crispin, T.S., Janetzki, A., Pirtle, E., Winwood-Smith, H.S., Angilletta, M.J., Chenoweth, S.F.,

744 Franklin, C.E., Halsey, L.G., Kearney, M.R., Portugal, S.J., Ortiz-Barrientos, D., 2019. The

745 origin and maintenance of metabolic allometry in animals. Nat. Ecol. Evol. 3, 598-603.

746 https://doi.org/10.1038/s41559-019-0839-9

747 Woods, H.A., Smith, J.N., 2010. Universal model for water costs of gas exchange by animals and

$748 \quad$ plants. Proc. Natl. Acad. Sci. U. S. A. 107, 8469-8474.

749 https://doi.org/10.1073/pnas.0905185107 
750 Xie, H., Lü, X., Zhou, J., Shi, C., Li, Y., Duan, T., Li, G., Luo, Y., 2017. Effects of acute

751 temperature change and temperature acclimation on the respiratory metabolism of the

752 snakehead. Turkish J. Fish. Aquat. Sci. 17, 535-542. https://doi.org/10.4194/1303-2712-

$753 \quad$ v17 $3 \quad 10$

754 Žagar, A., Carretero, M.A., Marguč, D., Simčič, T., Vrezec, A., 2018. A metabolic syndrome in 755 terrestrial ectotherms with different elevational and distribution patterns. Ecography (Cop.).

756 41, 1728-1739. https://doi.org/10.1111/ecog.03411

757 Zari A., T., 1996. Seasonal metabolic compensation in the fringe-toed lizard, acanthodactylus 758 boskianus (reptilia: lacertidae). J. Therm. Biol. 145-150.

759 
Table 1. Descriptive statistics (mean and SE) for body mass and body size (SVL) of aspic vipers maintained in three different thermal conditions during immature life stage (birth to 47 months post-birth) and kept in common environmental conditions during adult life stage (49 and 85 months post-birth).

\begin{tabular}{|c|c|c|c|c|}
\hline & & \multicolumn{3}{|c|}{ Treatment group (sample size) } \\
\hline & & Warm (16) & Medium (16) & Cold (17) \\
\hline Body mass (g) & Time of measurement & & & \\
\hline \multirow[t]{5}{*}{ Females (24) } & Birth & $6.51 \pm 0.35$ & $7.12 \pm 0.38$ & $7.32 \pm 0.41$ \\
\hline & 19 months post-birth & $17.86 \pm 2.05$ & $22.97 \pm 1.7$ & $21.18 \pm 2.08$ \\
\hline & 47 months post-birth & $117.31 \pm 4.94$ & $125.89 \pm 4.66$ & $139.29 \pm 5.55$ \\
\hline & 49 months post-birth & $112.39 \pm 4.31$ & $126.40 \pm 4.74$ & $137.61 \pm 4.42$ \\
\hline & 85 months post-birth & $156.50 \pm 8.84$ & $172.41 \pm 7.02$ & $187.79 \pm 4.57$ \\
\hline \multirow[t]{5}{*}{ Males (25) } & Birth & $7.23 \pm 0.55$ & $7.12 \pm 0.24$ & $7.12 \pm 0.37$ \\
\hline & 19 months post-birth & $17.89 \pm 1.97$ & $17.62 \pm 1.54$ & $23.13 \pm 2.57$ \\
\hline & 47 months post-birth & $89.80 \pm 7.78$ & $77.89 \pm 3.93$ & $79.72 \pm 4.07$ \\
\hline & 49 months post-birth & $83.65 \pm 7.07$ & $76.26 \pm 3.85$ & $80.19 \pm 4.22$ \\
\hline & 85 months post-birth & $88.93 \pm 7.11$ & $79.62 \pm 4.69$ & $86.03 \pm 4.32$ \\
\hline Body size (cm) & Time of measurement & & & \\
\hline \multirow[t]{4}{*}{ Females (24) } & Birth & $17.91 \pm 0.49$ & $18.27 \pm 0.38$ & $18.2 \pm 0.49$ \\
\hline & 30 months post-birth & $41.28 \pm 1.46$ & $38.12 \pm 1.82$ & $36.22 \pm 2.42$ \\
\hline & 49 months post-birth & $52.36 \pm 1.18$ & $50.86 \pm 2.25$ & $50.5 \pm 3.02$ \\
\hline & 85 months post-birth & $55.22 \pm 1.2$ & $56.93 \pm 1.55$ & $58.44 \pm 1.23$ \\
\hline \multirow[t]{4}{*}{ Males (25) } & Birth & $18.09 \pm 0.46$ & $18.47 \pm 0.46$ & $18.7 .12 \pm 0.27$ \\
\hline & 30 months post-birth & $39.22 \pm 2.11$ & $36.5 \pm 2.11$ & $37.39 \pm 1.56$ \\
\hline & 49 months post-birth & $46.54 \pm 2.18$ & $45.48 \pm 2.18$ & $46.92 \pm 1.28$ \\
\hline & 85 months post-birth & $47.36 \pm 2.25$ & $46.89 \pm 2.25$ & $47.83 \pm 1.19$ \\
\hline
\end{tabular}


767 Table 2. Variance and covariance components for residual (Ve and COVe) and individual ( $\mathrm{V}_{\mathrm{I}}$ and

$\left.768 \mathrm{COV}_{\mathrm{I}}\right)$ intercept of centered and scaled standard metabolic rate (SMR) and total evaporative water

769 loss (TEWL) rates in aspic vipers after controlling for trait-specific allometric relationships with

770 body mass and treatment-specific changes through time. Estimates are from the posterior-

771 distribution of (co)variance matrices.

772

\begin{tabular}{lll}
\hline Trait & Variance component & Estimate $\pm \mathbf{9 5 \%}$ CI \\
\hline SMR & Ve & $0.400[0.33,0.482]$ \\
& $\mathrm{V}_{\mathrm{I}}$ & $0.252[0.107,0.415]$ \\
\hline TEWL & $\mathrm{Ve}$ & $0.569[0.463,0.671]$ \\
& $\mathrm{V}_{\mathrm{I}}$ & $0.217[0.08,0.367]$ \\
\hline SMR-TEWL & $\mathrm{COVe}$ & $0.286[0.217,0.363]$ \\
& $\mathrm{COV}_{\mathrm{I}}$ & $0.168[0.048,0.324]$
\end{tabular}

773

774 
775 Table 3. Degree of metabolic and water loss compensation induced by acclimation response (\%

776 differences between values predicted by a null model and calculated DEEi and DEWL values) of

777 each treatment group at each session. P values are from t-test comparing DEEi and DEWL

778 predicted by a null model with calculated DEEi and DEWL. Because the acclimation response in

779 SMR did not persist at adulthood in the common garden (49 and 85 months post-birth, see Fig. 2A),

780 we estimated the degree of metabolic compensation only at 19 and 47 months post-birth.

781

\begin{tabular}{llll}
\hline Time of measurement & Treatment group & Degree of metabolic compensation \\
\hline 19 months post-birth & Warm & $38.36 \%$ & $\mathrm{P}<0.001$ \\
& Medium & $9.34 \%$ & $\mathrm{P}=0.26$ \\
& Cold & $0.21 \%$ & $\mathrm{P}=0.98$ \\
\hline 47 months post-birth & Warm & $42.21 \%$ & $\mathrm{P}<0.001$ \\
& Medium & $28.50 \%$ & $\mathrm{P}<0.001$ \\
& Cold & $5.42 \%$ & $\mathrm{P}=0.61$ \\
\hline Time of measurement & Treatment group & Degree of water loss compensation \\
\hline 47 months post-birth & Warm & $36.36 \%$ & $\mathrm{P}<0.001$ \\
& Medium & $13.46 \%$ & $\mathrm{P}=0.15$ \\
& Cold & $24.24 \%$ & $\mathrm{P}<0.001$ \\
\hline 49 months post-birth & Warm & $27.67 \%$ & $\mathrm{P}=0.05$ \\
& Medium & $14.92 \%$ & $\mathrm{P}=0.14$ \\
& Cold & $32.72 \%$ & $\mathrm{P}<0.001$ \\
\hline 85 months post-birth & Warm & $49.48 \%$ & $\mathrm{P}<0.001$ \\
& Medium & $16.26 \%$ & $\mathrm{P}=0.22$ \\
& Cold & $23.97 \%$ & $\mathrm{P}=0.08$ \\
\cline { 2 - 3 } & & & \\
& & & \\
& & &
\end{tabular}

782 


\section{Figure captions}

784 Figure 1. A) Experimental design and variables collected through time. 19 and 47 months post-

785 birth are during the time in which vipers were exposed to thermal treatments, while sessions 49 and

78685 months post-birth are during the time in which vipers were in a common garden. $\mathrm{VO}_{2}$ is the

787 oxygen consumption (standard metabolic rate); TEWL is Total Evaporative Water Loss; SVL is

788 Snout-Vent Length; BM is Body Mass. B) Daily temperature $\left({ }^{\circ} \mathrm{C}\right)$ cycle applied for each thermal

789 treatment (warm, medium, cold).

790 Figure 2. Effect of the thermal treatments on the residual standard metabolic rate (log transformed

$791 \mathrm{VO}_{2}$ provided in $\mathrm{mL}$ per hour, A) and residual total evaporative water loss (log transformed TEWL

792 provided in mg per hour, B) measured at different time points through lifetime (see Figure 1) at

$79320^{\circ} \mathrm{C}$ and $30^{\circ} \mathrm{C}$ body temperatures. Residual SMR and TEWL scores were calculated from a log-log

794 regression of individual raw values with body mass and thus accounts for ontogenetic and inter-

795 individual variation in body mass. Error bars represent SE. The * sign and different letters indicate

796 significant differences among treatment groups based on Tukey post-hoc tests of contrasts

797 performed A) in each treatment and temperature group, B) on average for all treatment and

798 temperature group. $\mathrm{ns}=$ not significant

799 Figure 3. A) Residual total evaporative water loss (log transformed TEWL provided in mg per 800 hour) against residual standard metabolic rate (log transformed $\mathrm{VO}_{2}$ provided in $\mathrm{mL}$ per hour) for 801 all aspic vipers across all lifetime stages, treatments and temperatures. B) Best linear unbiased 802 predictors (BLUP) of residual $\mathrm{VO}_{2}$ for the random effect of identity as function of BLUP for TEWL

803 for all aspic vipers with concurrent measurements of $\mathrm{VO}_{2}$ and TEWL at the same lifetime stage.

804 BLUPs and associated standard errors were extracted from univariate mixed models.

805 Figure 4. Daily expenditure during inactivity (DEEi), A) and daily total evaporative water loss 806 (DEWL), B) calculated at different time points through lifetime (see Figure 1A) for each treatment 807 group. White points represent mean values $( \pm \mathrm{SE})$ of calculated DEEi and DEWL. Black points 
808 represent single values of DEEi and DEWL predicted by a null model. Arrows symbolize the

809 degree of metabolic or water loss compensation. Because the acclimation response in SMR did not

810 persist at adulthood in the common garden (49 and 85 months post-birth, see Figure $2 \mathrm{~A}$ ), we

811 calculated and predicted DEEi only at 19 and 47 months post-birth. 


\section{Figure 1}

A

Daily temperature cycles

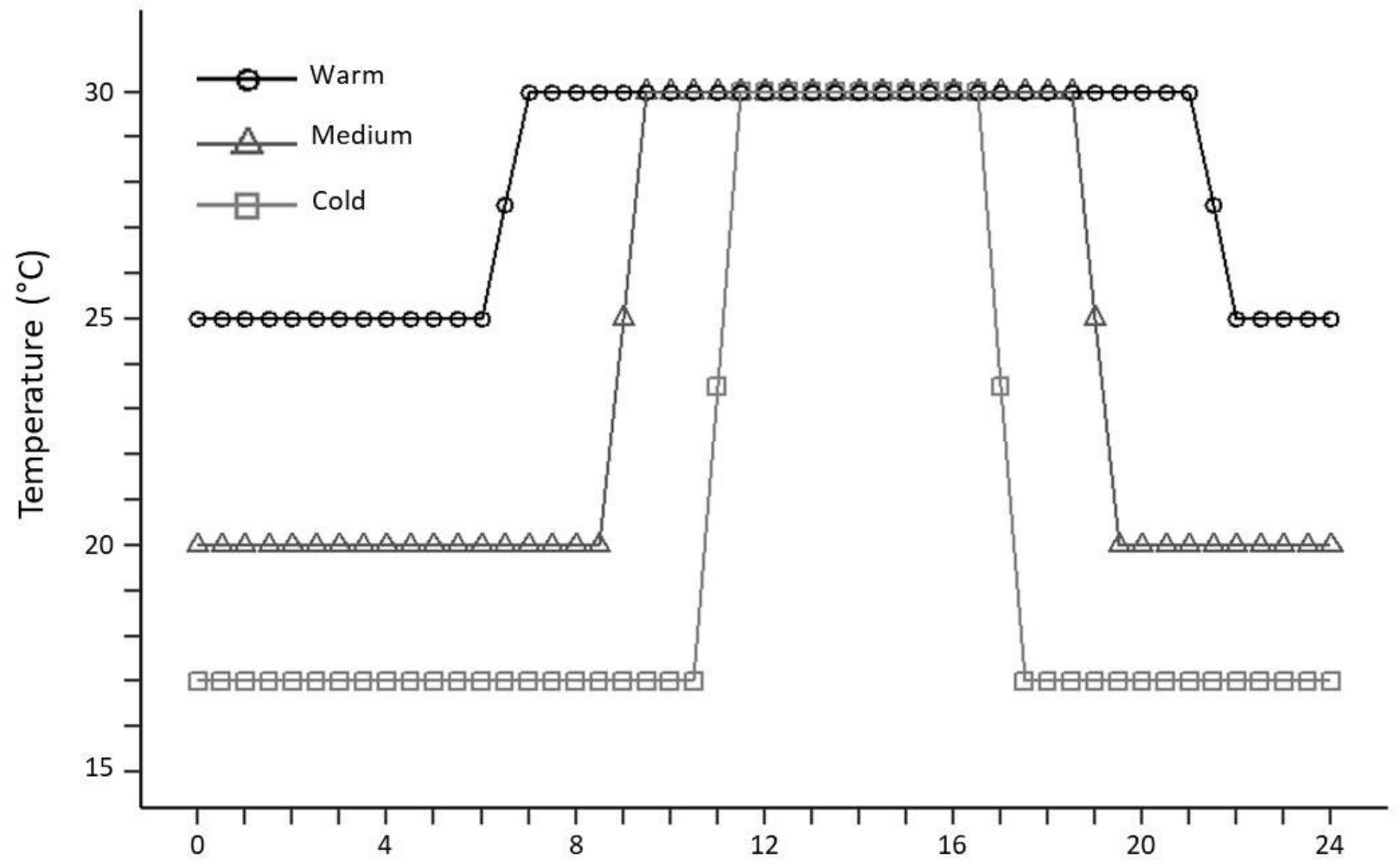

Hours

B

Experimental conditions :

Timeline :

Measured variables:

Life stage :

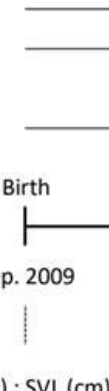

$\mathrm{BM}(\mathrm{g}) ; \mathrm{SVL}(\mathrm{cm})$
Treatment

Warm $(n=16) \mid$ Medium $(n=16) \mid$ Cold $(n=17)$

19 months post-birth $\quad 30$ months post-birth
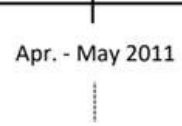

$\mathrm{BM}(\mathrm{g})$

$\mathrm{VO}_{2}\left(\mathrm{ml} \cdot \mathrm{h}^{-1}\right)$

Mar. 2012

SVL $(\mathrm{cm})$

\section{Common garden
Medium (all 49 individuals)}

49 months post-birth

85 months post-birth

$\mathrm{VO}_{2}\left(\mathrm{ml}^{\mathrm{h}} \mathrm{h}^{-1}\right)$ TEWL $\left(\mathrm{mg}^{-1} \mathrm{~h}^{-1}\right)$
BM (g)

Aug. 2013

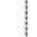

(1)

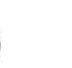

$\mathrm{VO}_{2}\left(\mathrm{ml} \cdot \mathrm{h}^{-1}\right)$
TEWL $\left(\mathrm{mg} \cdot \mathrm{h}^{-1}\right)$

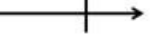

Oct.- Nov. 2016

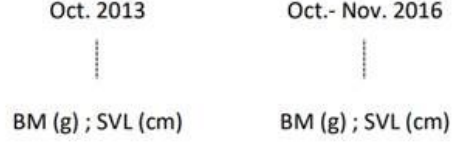

$\mathrm{VO}_{2}\left(\mathrm{ml}^{\mathrm{h}} \mathrm{h}^{-1}\right)$ TEWL (mg. $\mathrm{h}^{-1}$ ) 
814 Figure 2

A Trial temperature $\left({ }^{\circ} \mathrm{C}\right) \quad 020$

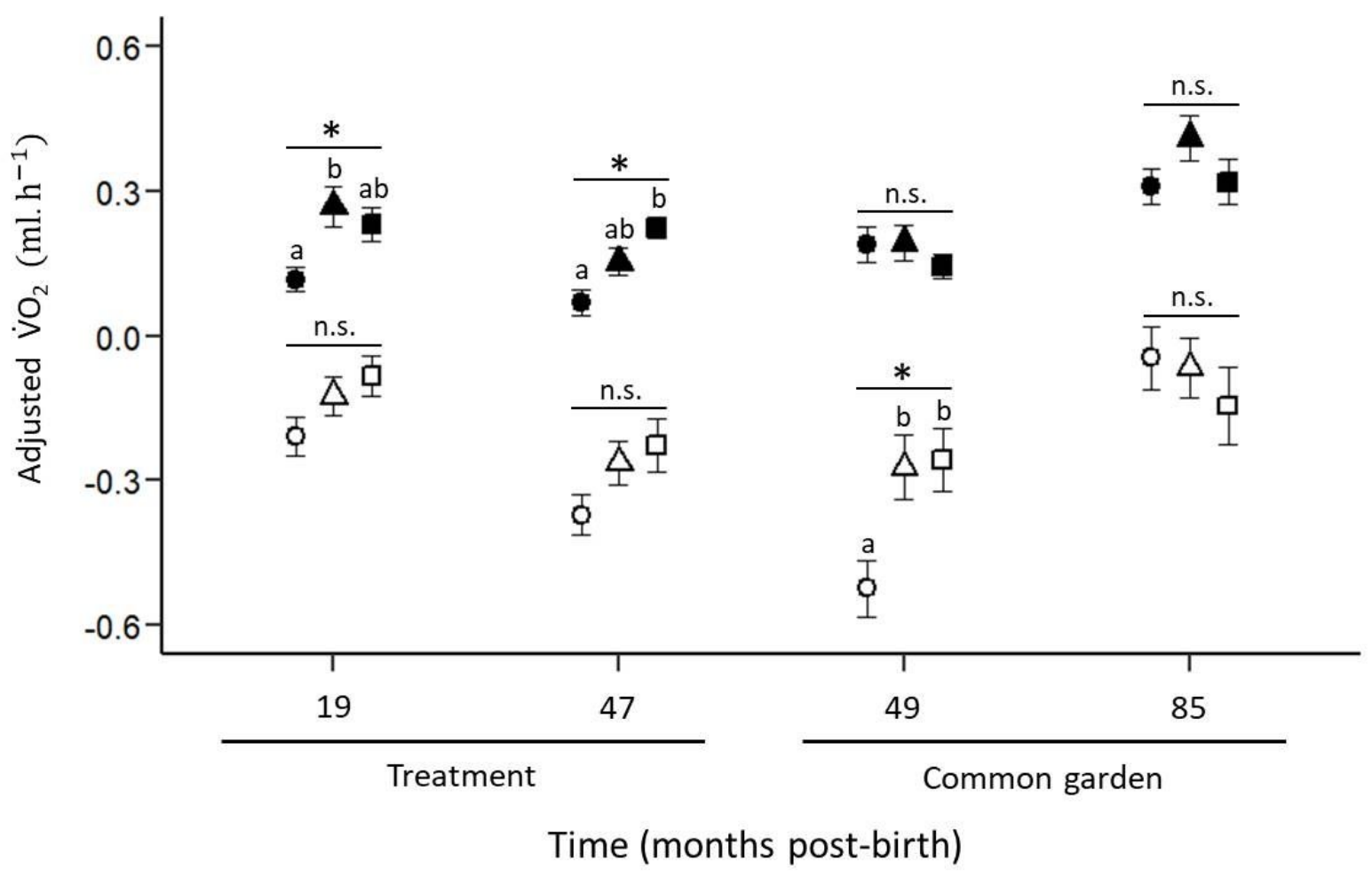

B

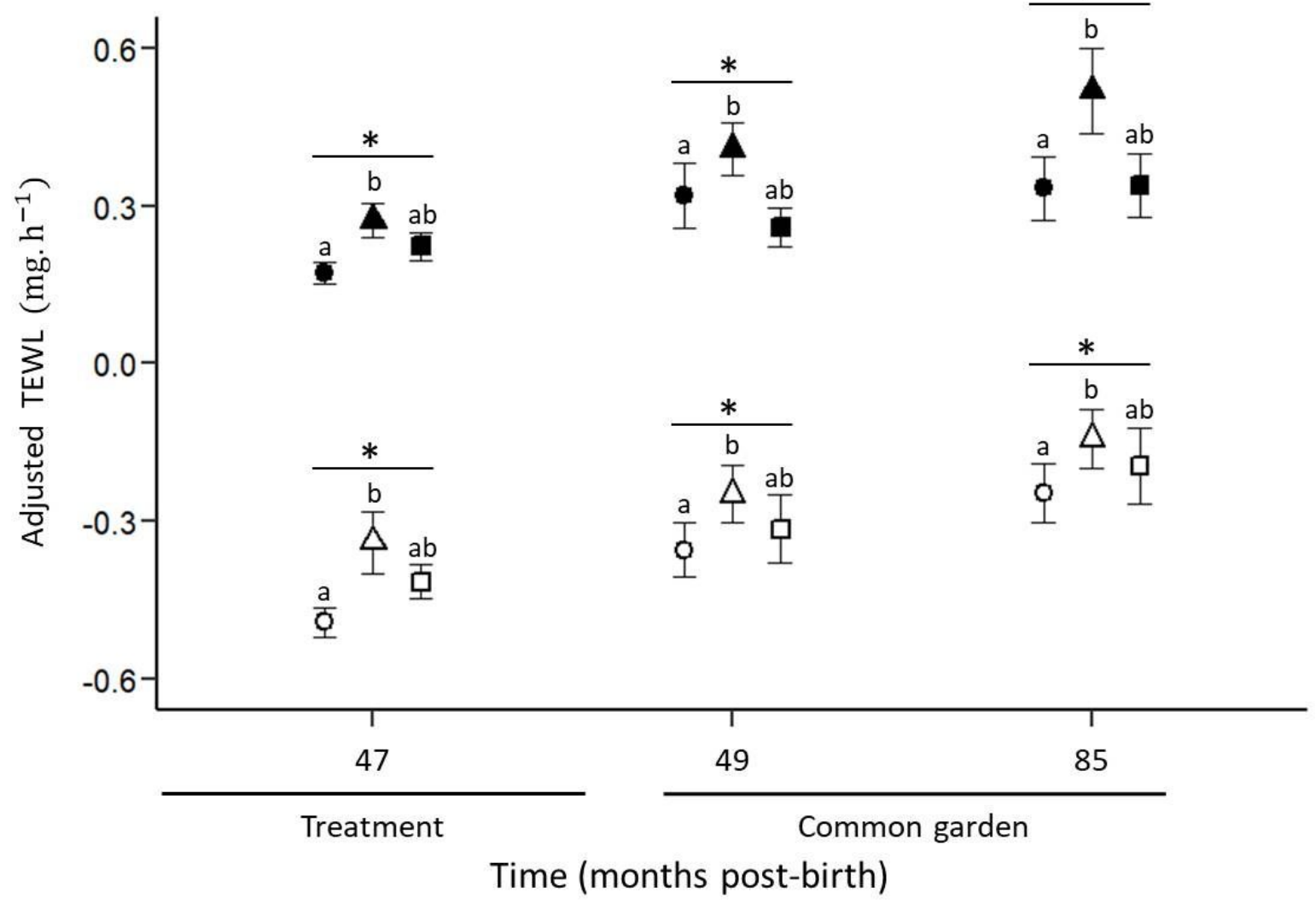


816 Figure 3
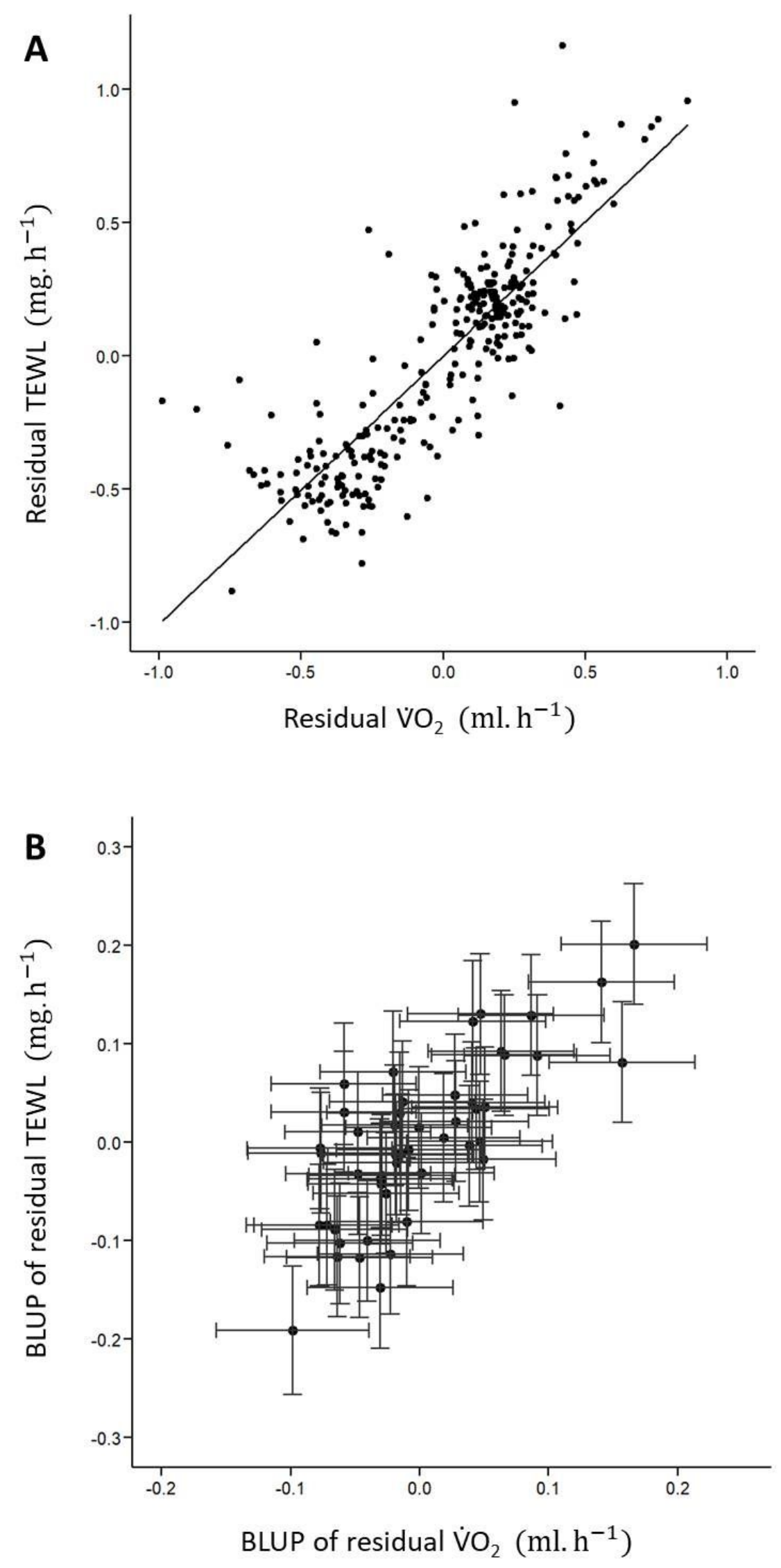


\section{$818 \quad$ Figure 4}

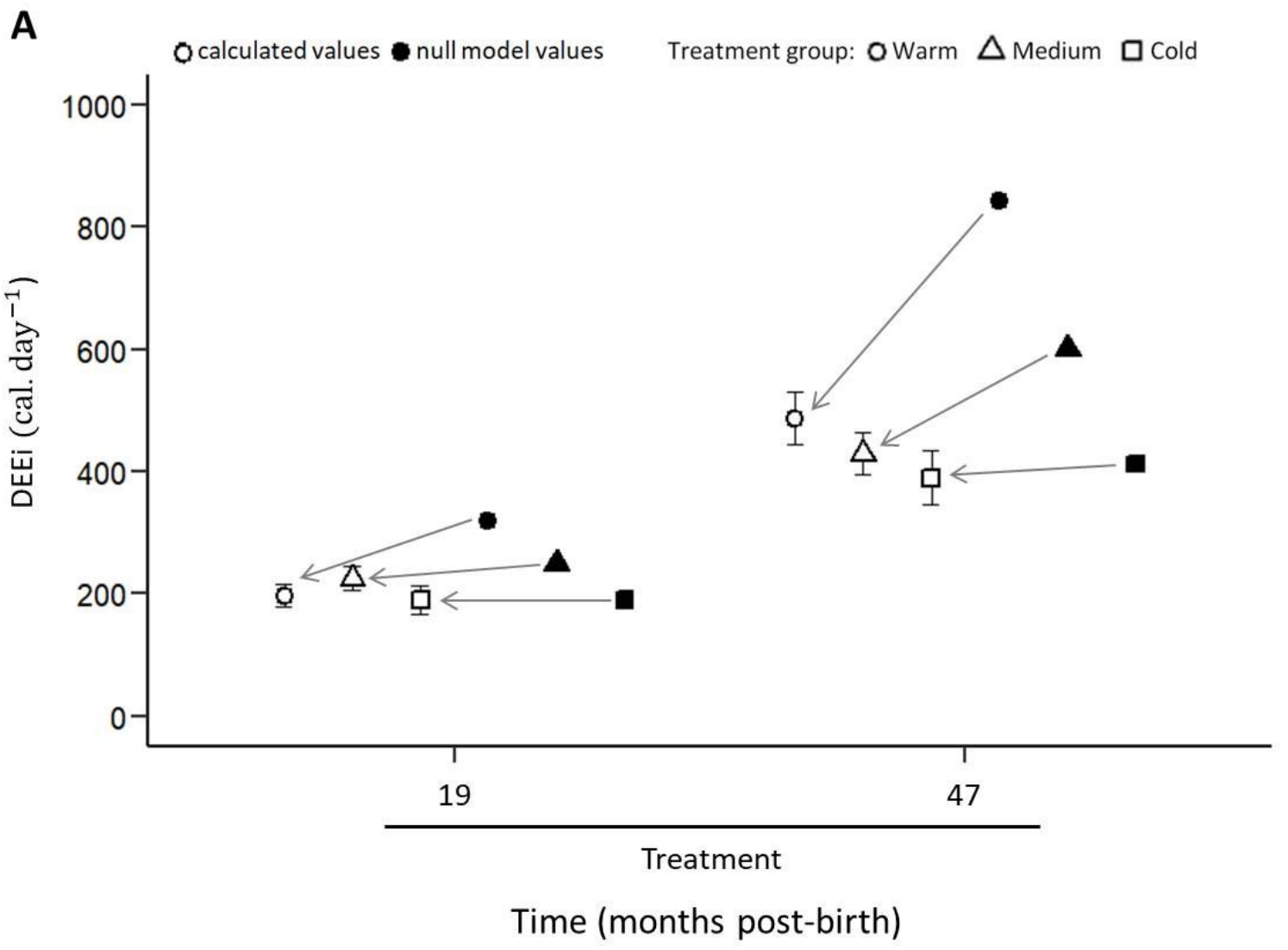

B

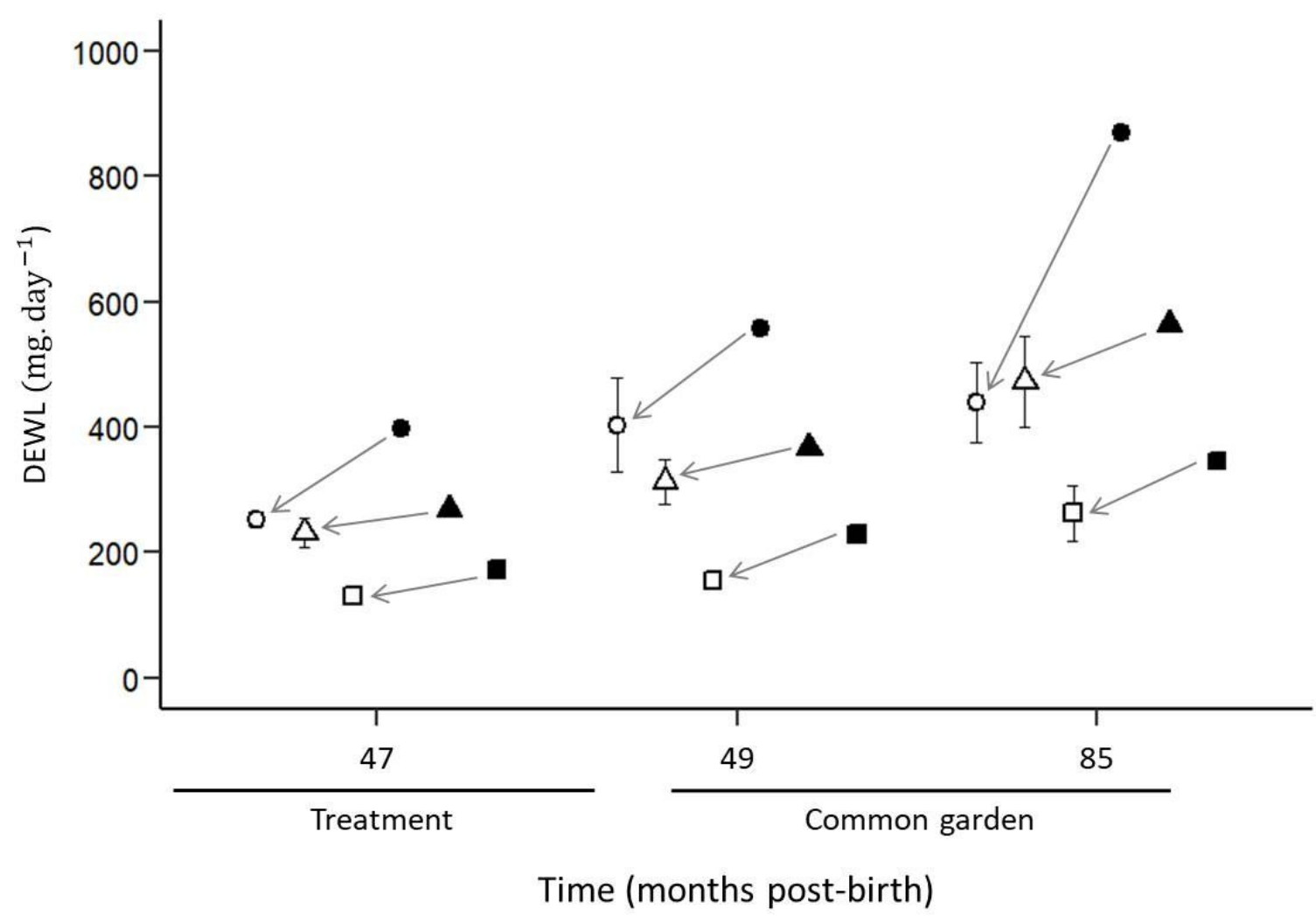

819 